\title{
The Landscape of Circular RNA Expression Profiles in Papillary Thyroid Carcinoma Based on RNA Sequencing
}

\author{
Xiabin Lan ${ }^{a, b} \quad$ Jiajie Xua ${ }^{a, b} \quad$ Chao Chen ${ }^{a, b} \quad$ Chuanming Zheng ${ }^{a, b}$ \\ Jiafeng Wang ${ }^{a, b}$ Jun Cao ${ }^{b}$ Xuhang Zhu ${ }^{a, b}$ Minghua Ge $e^{a, b}$

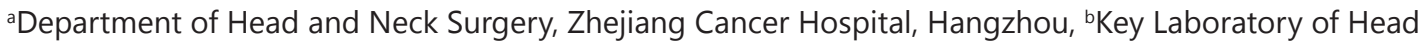 \\ \& Neck Cancer Translational Research of Zhejiang Province, Institute of Cancer Research, Zhejiang \\ Cancer Hospital, Hangzhou, China
}

\section{Key Words}

Circular RNA • MicroRNA • Papillary thyroid carcinoma • RNA sequencing

\begin{abstract}
Background/Aims: Papillary thyroid carcinoma (PTC) is the most common type of thyroid cancer. However, the molecular mechanisms responsible for its tumorigenesis and progression remain largely unknown. Circular RNA (circRNA) is a novel type of noncoding RNA that can serve as an ideal biomarker due to its stability. Recent evidence suggests that circRNAs play important roles in tumorigenesis. This study aims to investigate circRNA expression profiles and their potential biological functions in PTC. Methods: High-throughput RNA sequencing was used to assess circRNA expression profiles in PTC, and quantitative real-time polymerase chain reaction (qRT-PCR) was used to validate dysregulated circRNAs. Receiver operating characteristic (ROC) curves were generated to evaluate the diagnostic value of circRNAs for PTC. Gene Ontology (GO) and Kyoto Encyclopedia of Genes and Genomes (KEGG) pathway enrichment analyses were employed to determine the biological functions of differentially expressed circRNAs. Bioinformatic analyses were applied to predict interactions between circRNAs and microRNAs (miRNAs), and a circRNA-miRNA-mRNA network was constructed using Cytoscape software. Results: We identified a number of differentially expressed circRNAs in PTC tissues compared with paired normal thyroid tissues, with chr5:160757890160763776-, chr12:40696591-40697936+, chr7:22330794-22357656-, and chr21:1638666516415895 - being upregulated, and chr7:91924203-91957214+, chr2:179514891-179516047-, chr9:16435553-16437522-, and chr22:36006931-36007153- being downregulated. These findings were confirmed by $\mathrm{QRT}-\mathrm{PCR}$, and ROC curves indicated that they can serve as potential biomarkers for PTC. GO and KEGG pathway analyses showed that some of these circRNAs are related to cancers. Additionally, bioinformatic analyses revealed a potential competingendogenous-RNA-regulating network among circRNAs, miRNAs, and mRNAs. Conclusions:
\end{abstract}

X. Lan and J. Xu contributed equally to this work.

Minghua Ge

KARGER
Department of Head and Neck Surgery, Zhejiang Cancer Hospital

No. 1 Banshandong Road, Gongshu District, Hangzhou, Zhejiang 310022 (China)

Tel. +86 0571 88128102, Fax +86 0571 88122508, E-Mail gemingh@163.com 
Our study results depict the landscape of circRNA expression profiles in PTC and also provide potential biomarkers for PTC. Further functional and mechanistic studies of these circRNAs may improve our understanding of PTC tumorigenesis.

(C) 2018 The Author(s)

Published by S. Karger AG, Basel

\section{Introduction}

The incidence of thyroid cancer has increased rapidly ( $>5 \%$ per year in both men and women) over the past several decades [1]. Accounting for more than $80 \%$ of all cases, papillary thyroid carcinoma (PTC) represents the major histopathologic type of thyroid cancer [2]. Although PTC is usually curable, with a 5-year survival of over 95\%, PTCs with more aggressive phenotypes, such as extrathyroidal extension, multifocal tumors, and lymph node and distant metastases, can dedifferentiate into more lethal thyroid cancers. These more aggressive PTCs may not be efficiently managed by traditional treatments, such as surgery, thyroid hormone therapy, or radioactive iodine therapy [3]. Therefore, it is necessary to identify individuals with a genetic predisposition toward PTC and those with potentially more aggressive types of PTC who may need individual treatment strategies or novel therapeutic approaches.

Some genetic alterations associated with PTC pathogenesis (e.g., BRAF, RAS, and RET/ PTC mutations) have been evaluated as diagnostic or prognostic markers [2, 3], though the molecular markers reported thus far are mainly protein-coding genes. However, it is estimated that less than $2 \%$ of the human genome encodes proteins, and the majority of the human transcriptome consists of noncoding RNAs. Accumulating evidence demonstrates that noncoding RNAs, such as microRNAs (miRNAs) and long noncoding RNAs (lncRNAs), play an important regulatory role in many physiological and pathophysiological processes. More recently, a novel class of noncoding RNA termed circular RNA (circRNA) has become a hot topic in RNA research. In contrast to linear RNAs that are terminated with $5^{\prime}$ caps and $3^{\prime}$ tails, circRNAs are characterized by covalently closed continuous loops with neither $5^{\prime}-3^{\prime}$ polarity nor a polyadenylated tail [4]. circRNAs are commonly found in mammalian cells and can regulate gene expression at the transcriptional or post-transcriptional level by acting as miRNA sponges or by interacting with other molecules [5]. Although circRNAs were discovered decades ago, studies about their functions have emerged only recently. Moreover, circRNAs appear to be expressed in tissue- and developmental-stage-specific manners, which indicates that they function in many physiological and pathophysiological processes $[6,7]$. Similar to miRNAs and lncRNAs, researchers have also found that circRNAs are related to many diseases, such as atherosclerosis, nervous system disorders, and a variety of cancers [8-11]. circRNAs are stable in cells because they are resistant to exonucleases; thus, circRNAs may constitute ideal biomarkers for cancer diagnosis [12]. Regardless, identifying dysregulated circRNAs in different cancers and elucidating their functions remains an ongoing process in cancer research.

To date, little is known about the associations between circRNAs and PTC. Therefore, in the current study, we screened the circRNA profiles of PTC patients using high-throughput RNA sequencing (RNA-Seq) and validated dysregulated circRNAs in PTC tissues by quantitative real-time polymerase chain reaction (qRT-PCR). Bioinformatic analysis was then performed to predict their potential roles in PTC and lay a foundation for future studies of circRNAs that are related to PTC.

\section{Materials and Methods}

Patient samples

A total of 90 pairs of PTC and adjacent normal thyroid tissues were collected from patients at the Department of Head and Neck Surgery of the Zhejiang Cancer Hospital between September 2016 and August 2017 (Tables 1 and 2). The diagnosis of PTC and the adjacent normal tissues was independently 
Table 1. Clinicopathologic characteristics of patients from whom samples were obtained for circRNA sequencing

\begin{tabular}{lccc}
\hline Patient No. & Sex & Age & TNM (AJCC, 7th, ed. 2010) \\
\hline 1 & female & 51 & T4aN1bM0, IVa \\
2 & female & 55 & T3N1aM0, III \\
3 & female & 57 & T4aN1aM0, IVa \\
\hline
\end{tabular}

confirmed by two pathologists (Fig. 1). The patients did not receive any chemotherapy or radiotherapy prior to surgical resection. All tissue samples were snap-frozen and stored in liquid nitrogen until further experiments were performed. Written informed consent was obtained from all patients, and this study was approved by the ethics committee of the Zhejiang Cancer Hospital, Hangzhou, China.

\section{RNA extraction and quality} control

Total RNA was isolated from each tissue sample using TRIzol reagent (Life Technologies, Carlsbad, CA, USA) following the manufacturer's instructions. The quality of the RNA samples was determined by OD 260/280 assessment using a NanoDrop ND-2000 instrument (Thermo Fisher Scientific, Waltham, MA, USA). RNA integrity was determined by denaturing agarose gel electrophoresis.

circRNA sequencing and bioinformatic analysis

Total RNA from each sample was used to prepare circRNA sequencing libraries according to a previously described protocol [13]. Paired-end reads were harvested from an Illumina HiSeq 4000 sequencer, and quality control was performed with a Q30. After 3' adapter trimming and low-quality read removal with cutadapt software (v1.9.3), high-quality reads were aligned to the reference genome/transcriptome with STAR software [14]. circRNAs were detected and annotated with DCC software [15] using two public circRNA databases: circBase [16] and circ2Traits [17]. The junction read counts were normalized, and differentially expressed circRNAs were determined using the edge $\mathrm{R}$ package of $\mathrm{R}$ software [18]. circRNAs that exhibited fold changes (FCs) $\geq 2.0$ with $P$ values $<0.05$ were considered to be significantly differentially expressed. Gene Ontology (GO) and Kyoto Encyclopedia of Genes and Genomes (KEGG) pathway enrichment analyses were performed for the host genes of the differentially expressed circRNAs. circRNA-miRNA interactions and miRNA targets were predicted with popular target prediction software $[19,20]$, and a network was constructed with Cytoscape software [21].

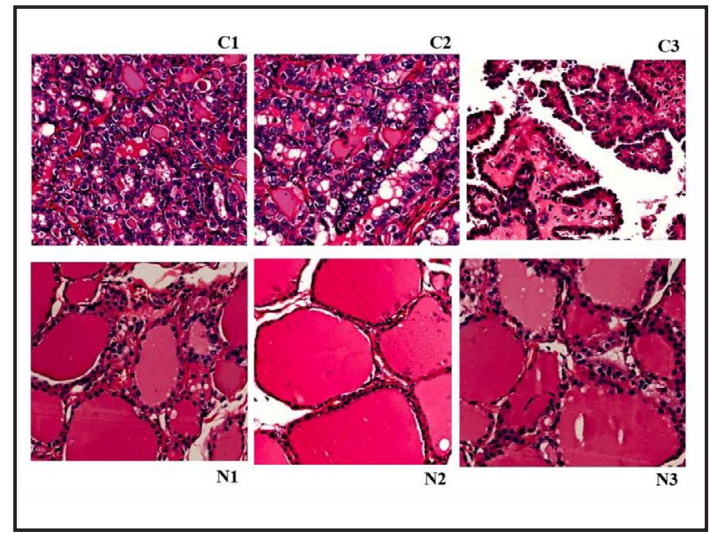

Fig. 1. Representative hematoxylin-eosin-stained images of PTC (C1-C3) and adjacent normal tissues (N1-N3). 
qRT-PCR validation

Qualified total RNA was reverse transcribed to synthesize cDNA using PrimeScript RT Master Mix (Perfect Real Time, TaKaRa, Shiga, Japan) and then analyzed by qRT-PCR with SYBR Premix Ex Taq II (Tli RHaseH Plus, TaKaRa) and a LightCycler 480 system (Roche, Basel, Switzerland). Glyceraldehyde 3-phosphate dehydrogenase (GAPDH) was used as the internal control. The primers used for qRT-PCR analysis were as follows: chr5:160757890-160763776- (F 5'-TGGGGTGCTTTGTCTTCGTT-3', R 5'-TCAGTTGTGTATCCATCTTGTTGAC-3'), chr12:40696591-40697936+ (F 5'-ACTGAAAGAGCTGCTATGCCT-3', R 5'-AAGACCGCAAGTGTGGAAGA-3'), chr7:22330794-22357656- (F 5'-TGCCTCTCATTCCTGCCAGA-3', R 5'-TCTTGATAGAGTCGCAGATGTTAGA-3'), chr21:16386665-16415895(F 5'-TCTGAAGACTCCGGATGACA-3'， R 5'- GTGTGCATCTTCTGGCTGTG-3'), chr7:91924203-91957214+ (F 5'-TCGAGCTCATGAATGTGAAAGATG-3', $\mathrm{R} \quad 5^{\prime}$-GTGCTTTACGGAATTTGGTGGT-3'), chr2:179514891179516047- (F 5'-AAGCCAGTTCCTGTTGCAGA-3', R 5'-TTTGCTGGTGGGACTTCTGG-3'), chr9:1643555316437522- (F 5'-CGAAGCCGAGACAGGATGC-3', R 5'-GCCTGCCCTTCTTTCTCCTG-3'), chr22:3600693136007153- (F 5'-TACCTGGAGGCTCTTTAAGGGT-3', R 5'-CAGACGCCTTCATCTCGTCC-3') and GAPDH (F $5^{\prime}$-CAGGAGGCATTGCTGATGAT-3', R 5'-GAAGGCTGGGGCTCATTT-3'). The relative expression level of each circRNA was calculated using the $2^{-\Delta \Delta c t}$ method.

\section{Statistical analyses}

Statistical analyses were performed using Prism 6 (GraphPad Software, La Jolla, CA) and SPSS 19.0 (IBM, Chicago, IL, USA). $P<0.05$ was considered statistically significant. Differences between groups were assessed using Student's $t$ test or Wilcoxon's rank-sum test, as appropriate. Receiver operating characteristic (ROC) curves were established to evaluate the diagnostic value of circRNAs for PTC.

\section{Results}

\section{Overview of circRNA profiles}

A total of 9, 103 circRNAs were identified by RNA-Seq in three pairs of PTC and normal thyroid tissue samples. Statistically significant differentially expressed circRNAs between the two groups are displayed through FC filtering (Fig. 2A, B). The heat map

Fig. 2. Overview of circRNA profiles (CA: PTC tissues; N: normal thyroid tissues; chr: chromosome). (A) Volcano plot of differentially expressed circRNAs in PTC. The vertical green lines correspond to two-fold increased and decreased expression, and the horizontal green line represents $P=0.05$. The red points represent circRNAs that were differentially expressed with statistical significance. (B) The scatter plot presents the circRNA expression variations between the PTC and paired adjacent normal thyroid tissues. The
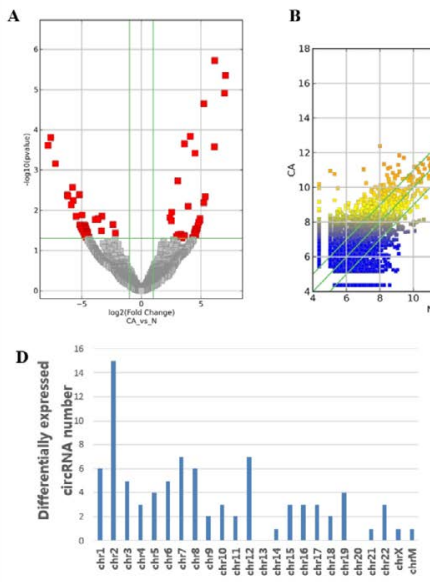
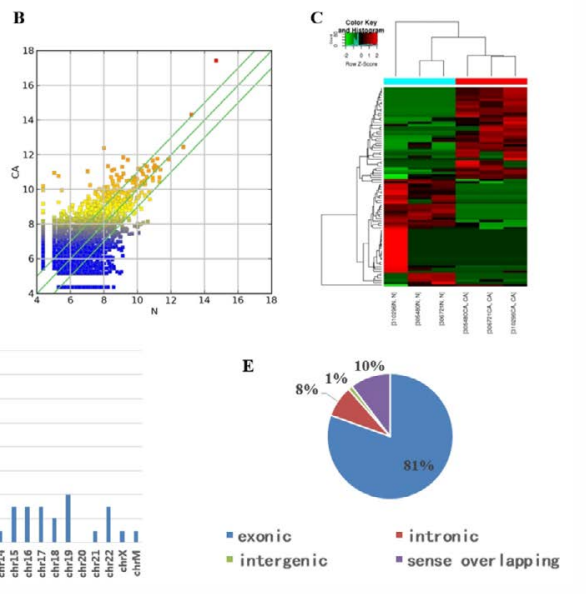
values of the $\mathrm{x}$ and $\mathrm{y}$ axes are the normalized signal values of two groups of samples (log2 scaled). The circRNAs above the top green line and below the bottom green line indicate greater than 2 -fold change between the two groups of samples. (C) Hierarchical cluster analysis of differentially expressed circRNAs. The red and green colors indicate high and low expression, respectively. (D) Chromosomal distributions of differentially expressed circRNAs. (E) Classification of differentially expressed circRNAs based on genomic origin. 
shown in Fig. 2C illustrates the circRNA expression profiles that were distinguishable between the cancer and normal tissues. Consequently, 87 circRNAs were detected as differentially expressed by FC $\geq 2.0$ and $P<0.05$, of which 41 were upregulated and 46 downregulated; the numbers of upregulated circRNAs and downregulated circRNAs were approximately equal. As presented in Fig. 2D, the transcription of differentially regulated circRNAs was found to be widely distributed among all chromosomes except chr13 and chr20, with chr2, chr7, chr12, chr1, and chr8 being the top five. Among these differentially expressed circRNAs, 70 are exonic and seven intronic; one belongs to the group of intergenic circRNAs, and nine are transcribed from overlapping areas (Fig. 2E). Moreover, 33 wereidentified as new circRNAs that have not been annotated in the circBase or circ2Traits database. The top 20 up- and downregulated circRNAs are listed in Table 3 according to FC.
Table 3. Top 20 differentially expressed circRNAs in PTC (FC, fold change)

\begin{tabular}{|c|c|c|c|c|c|c|c|c|}
\hline circRNA ID & $\log \mathrm{FC}$ & P Value & regulation & chrom & circBase ID & Gene Name & Catalog & length \\
\hline chr5:160757890-160763776- & 7.0594638 & $4.455 \mathrm{E}-06$ & up & chr5 & & GABRB2 & exonic & 536 \\
\hline chr12:40696591-40697936+ & 6.9757106 & $1.224 \mathrm{E}-05$ & up & chr12 & hsa_circ_0025887 & LRRK2 & exonic & 281 \\
\hline chr12:94562929-94580249+ & 6.1494217 & $1.873 \mathrm{E}-06$ & up & chr12 & & PLXNC1 & exonic & 377 \\
\hline chr7:22347958-22357656- & 6.1289577 & 0.0002622 & up & chr7 & & RAPGEF5 & exonic & 449 \\
\hline chr7:22330794-22357656- & 5.3544779 & 0.0045872 & up & chr7 & hsa_circ_0001681 & RAPGEF5 & exonic & 516 \\
\hline chr15:94899366-94928754+ & 5.2425776 & $2.232 \mathrm{E}-05$ & up & chr15 & & MCTP2 & exonic & 783 \\
\hline chr19:57088081-57089646+ & 5.2420838 & 0.0064241 & up & chr19 & & ZNF470 & intronic & 1566 \\
\hline chr15:94910834-94928754+ & 4.9201652 & 0.0178311 & up & chr15 & & MСТР2 & exonic & 487 \\
\hline chr22:23243156-23260373+ & 4.9161654 & 0.0162592 & up & chr22 & & & intergenic & 17218 \\
\hline chr11:89165952-89185063- & 4.8185828 & 0.0196645 & up & chr11 & hsa_circ_0023990 & NOX4 & exonic & 395 \\
\hline chr2:203817282-203826149+ & 4.741227 & 0.0235486 & up & chr2 & hsa_circ_0003747 & CARF & exonic & 526 \\
\hline chr5:170305101-170323119+ & 4.6828141 & 0.0278566 & up & chr5 & hsa_circ_0001554 & RANBP17 & exonic & 471 \\
\hline chr4:174153603-174169591+ & 4.6395841 & 0.0308816 & up & chr4 & & GALNT7 & sense overlapping & 15989 \\
\hline chr18:20570900-20581692+ & 4.5742248 & 0.0328106 & up & chr18 & hsa_circ_0047136 & RBBP8 & exonic & 1480 \\
\hline chr16:29845054-29845387+ & 4.5645496 & 0.0410921 & up & chr16 & hsa_circ_0000688 & MVP & exonic & 256 \\
\hline chr5:123974826-124036962- & 4.5432473 & 0.0294251 & up & chr5 & hsa_circ_0073716 & ZNF608 & exonic & 3626 \\
\hline chr8:18622959-18662408- & 4.5014113 & 0.000377 & up & chr8 & hsa_circ_0002111 & PSD3 & exonic & 538 \\
\hline chr2:9028148-9098771- & 4.4839511 & 0.0346522 & up & chr2 & hsa_circ_0008847 & MBOAT2 & exonic & 376 \\
\hline chr2:196544756-196545774+ & 4.4623798 & 0.0410323 & up & chr2 & hsa_circ_0057550 & SLC39A10 & exonic & 1019 \\
\hline chr6:32632627-32729488- & 4.4523293 & 0.0376792 & up & chr6 & & HLA-DQB1 & sense overlapping & 96862 \\
\hline chr22:36006931-36007153- & -7.833294 & 0.0002446 & down & chr22 & hsa_circ_0063050 & MB & exonic & 223 \\
\hline chr9:16435553-16437522- & -7.610093 & 0.0001563 & down & chr9 & hsa_circ_0086414 & BNC2 & exonic & 1970 \\
\hline chr2:179517185-179517463- & -7.2153 & 0.000688 & down & chr2 & & TTN & exonic & 168 \\
\hline chr2:179534319-179535022- & -6.211052 & 0.0041329 & down & chr2 & & TTN & exonic & 162 \\
\hline chr2:179545806-179546177. & -6.211052 & 0.0041329 & down & chr2 & & TTN & exonic & 168 \\
\hline chr2:179514891-179516047. & -6.169081 & 0.0044674 & down & $\operatorname{chr} 2$ & & TTN & exonic & 270 \\
\hline chr2:179594015-179594293- & -5.889572 & 0.007312 & down & chr2 & & TTN & exonic & 279 \\
\hline chr2:179545806-179546141- & -5.889572 & 0.007312 & down & chr2 & & TTN & intronic & 336 \\
\hline chr8:62531537-62566219- & -5.779114 & 0.0026795 & down & chr8 & hsa_circ_0084606 & ASPH & exonic & 654 \\
\hline chr8:41518948-41519459- & -5.722471 & 0.0056978 & down & chr8 & hsa_circ_0001792 & ANK1 & exonic & 201 \\
\hline chr5:137219073-137219280+ & -5.479514 & 0.0140916 & down & chr5 & & МYOT & exonic & 208 \\
\hline chr14:23873505-23900690- & -5.205989 & 0.0040941 & down & chr14 & & MYH7 & sense overlapping & 27186 \\
\hline chr2:179559556-179560107. & -5.167508 & 0.0229073 & down & chr2 & & TTN & intronic & 552 \\
\hline chr1:200816768-200822623+ & -5.167508 & 0.0229073 & down & chr1 & & CAMSAP2 & exonic & 2631 \\
\hline chr19:4511523-4511918- & -5.079222 & 0.0259603 & down & chr19 & & PLIN4 & sense overlapping & 396 \\
\hline chr2:179560592-179560759- & -5.079222 & 0.0259603 & down & $\operatorname{chr} 2$ & & TTN & intronic & 168 \\
\hline chr6:170846322-170858201- & -4.984135 & 0.0296471 & down & chr6 & hsa_circ_0078784 & PSMB1 & exonic & 427 \\
\hline chr18:6301902-6312055- & -4.975557 & 0.0131084 & down & chr18 & & L3MBTL4 & exonic & 217 \\
\hline chr2:168101672-168101821+ & -4.877317 & 0.0345266 & down & chr2 & & XIRP2 & intronic & 150 \\
\hline chr16:24979672-24981901- & -4.813434 & 0.0231379 & down & chr16 & hsa_c & ARHGAP17 & exonic & 263 \\
\hline
\end{tabular}

Table 4. Area under the curve (AUC) of qRT-PCR-validated circRNAs in the discovery and validation cohorts

\begin{tabular}{lcccccc}
\hline & \multicolumn{3}{c}{ Discovery cohort $(\mathrm{n}=44)$} & \multicolumn{3}{c}{ Validation cohort $(\mathrm{n}=43)$} \\
circRNA & AUC & $95 \%$ CI & P & AUC & $95 \%$ CI & P \\
\hline chr5:160757890-160763776- & 0.9566 & $0.9088-1.004$ & $<0.0001$ & 0.9502 & $0.8995-1.001$ & $<0.0001$ \\
chr12:40696591-40697936+ & 0.9476 & $0.9041-0.9911$ & $<0.0001$ & 0.9448 & $0.8966-0.9931$ & $<0.0001$ \\
chr7:22330794-22357656- & 0.9099 & $0.8433-0.9764$ & $<0.0001$ & 0.9186 & $0.8607-0.9765$ & $<0.0001$ \\
chr21:16386665-16415895- & 0.7200 & $0.6128-0.8272$ & 0.0003813 & 0.7309 & $0.6212-0.8407$ & 0.0002284 \\
chr7:91924203-91957214+ & 0.8763 & $0.8045-0.9481$ & $<0.0001$ & 0.8661 & $0.7922-0.9401$ & $<0.0001$ \\
chr2:179514891-179516047- & 0.8270 & $0.7343-0.9196$ & $<0.0001$ & 0.8253 & $0.7318-0.9189$ & $<0.0001$ \\
chr9:16435553-16437522- & 0.8192 & $0.7306-0.9078$ & $<0.0001$ & 0.8213 & $0.7319-0.9106$ & $<0.0001$ \\
chr22:36006931-36007153- & 0.9305 & $0.8796-0.9814$ & $<0.0001$ & 0.9348 & $0.8794-0.9902$ & $<0.0001$ \\
\hline
\end{tabular}

\section{Validation of circRNA expression}

Eight circRNAs (four were the most differentially expressed, and four were randomly chosen) were selected to verify the RNA-Seq in another 44 pairs of samples by qRT-PCR. The results revealed that expression of chr5:160757890-160763776-, chr12:4069659140697936+, chr7:22330794-22357656-, and chr21:16386665-16415895- was upregulated, whereas that of chr7:91924203-91957214+, chr2:179514891-179516047-, chr9:16435553-16437522-, and chr22:36006931-36007153- was downregulated (Fig. 3A). The qRT-PCR data were strongly consistent with the circRNA sequencing data (Fig. 3B), indicating the reliability of the RNA-Seq results.

\section{Diagnostic value of qRT-PCR-validated circRNAs}

To assess whether the identified circRNAs can serve as potential diagnostic biomarkers for PTC, we built ROC curves for qRT-PCR-validated circRNAs using both a discovery cohort $(n=44$, Fig. $4 \mathrm{~A})$ and a validation cohort $(n=43$, Fig. 4B). The results revealed that these 
circRNAs are promising biomarkers for identifying PTC, with consistent areas under the curve in both the validation and discovery cohorts (Table 4).

\section{GO analysis and pathway analysis of circRNA genes}

GO annotation revealed the host genes of the identified differentially expressed circRNAs, as presented in Fig. 5. The most significantly enriched GO terms in the biological process, cellular component, and molecular function categories were muscle system process (G0: 0003012, $P=1.42 \mathrm{E}-05$ ), $\mathrm{Z}$ disc (GO: 0030018, $P=1.33 \mathrm{E}-07$ ), and alpha-actinin binding (G0: 0051393, $P=1.11 \mathrm{E}-06$ ), respectively. The results of KEGG pathway analysis of the circRNA genes are presented in Fig. 6. The differentially expressed circRNA genes are mainly associated with autoimmune thyroid disease, viral myocarditis, allograft rejection, graft-versus-host disease, type I diabetes mellitus, epithelial cell signaling in Helicobacter pylori infection, PPAR signaling pathway, adherens junction, axon guidance, antigen processing and presentation, and herpes simplex infection.

miRNA prediction and circRNAmiRNA-mRNA network construction

Because circRNAs contain corresponding miRNA binding sites and can function as miRNA sponges, to evaluate their potential functions in PTC, we investigated miRNAs potentially associated with the identified differentially expressed circRNAs using miRNA target prediction software. The first five predicted miRNAs for the top three up- and downregulated circRNAs are listed in Table 5. For example, circRNA chr5:160757890-160763776- is predicted to harbor hsa-miR-3154, hsamiR-2392, hsa-miR-6833-5p, hsa-miR7114-5p, and hsa-miR-5004-5p, with different seed sequence matching types (i.e., 7 mer-m8, 8mer, 6mer, or imperfect; Fig. 7). Additionally, a circRNA-miRNA-

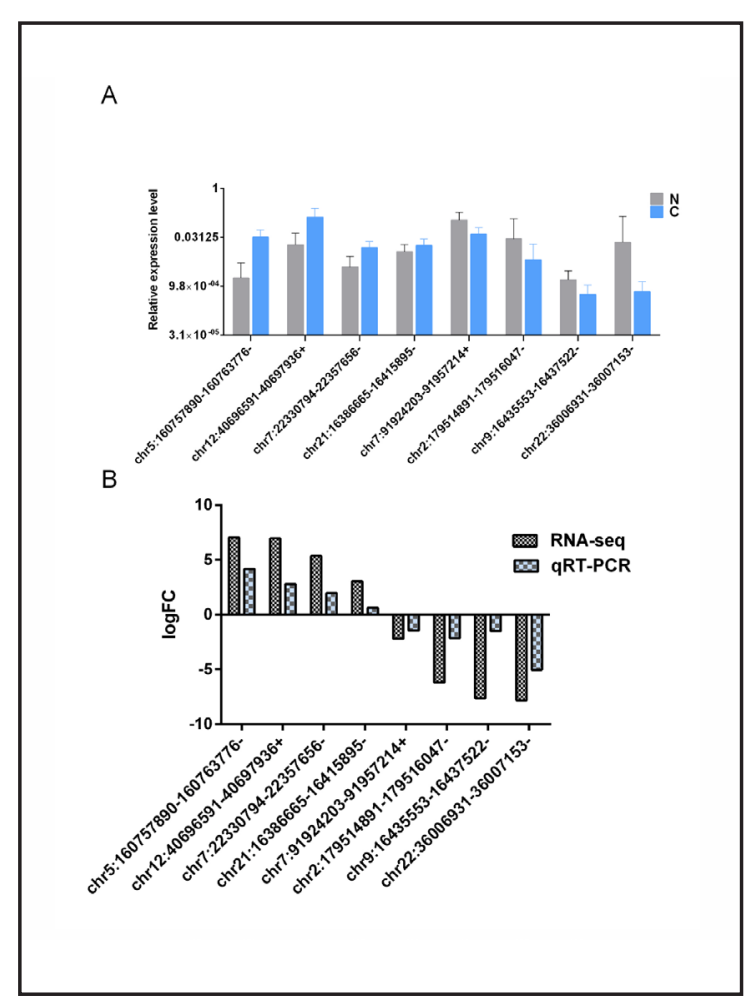

Fig. 3. qRT-PCR validation of eight differentially expressed circRNAs. (A) The relative expression levels of circRNAs in 44 PTC tissues and paired normal thyroid tissues (N: normal thyroid tissues; C: PTC tissues; $\mathrm{P}<0.05$ ). (B) Comparison of $\log 2$ fold changes (FCs) in circRNAs between RNA-Seq and qRT-PCR results.
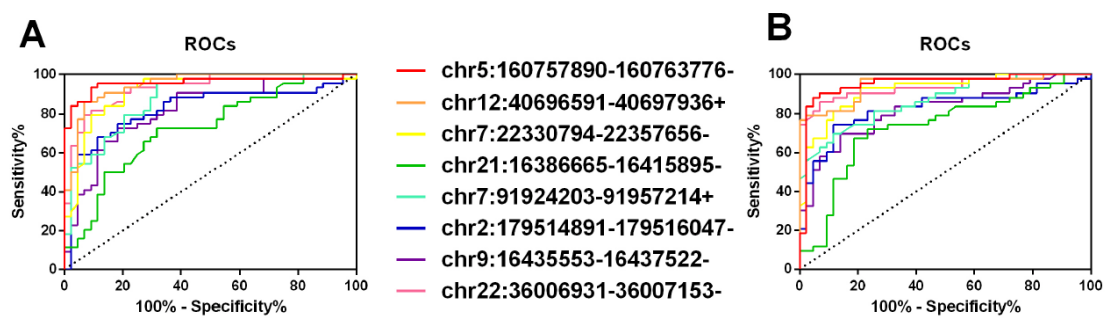

Fig. 4. Receiver operating characteristic (ROC) curves of eight qRT-PCR-validated circRNAs. (A) Discovery cohort $(n=44)$. (B) Validation cohort $(n=43)$. 
Table 5. Predicted miRNAs for the top three up- or downregulated circRNAs in PTC

\begin{tabular}{lccccc}
\hline circRNA & miRNA & miRNA & miRNA & miRNA & miRNA \\
\hline chr5:160757890-160763776- & hsa-miR-3154 & hsa-miR-2392 & hsa-miR-6833-5p & hsa-miR-7114-5p & hsa-miR-5004-5p \\
chr12:40696591-40697936+ & hsa-miR-648 & hsa-miR-6833-5p & hsa-miR-6505-5p & hsa-miR-5683 & hsa-miR-767-3p \\
chr12:94562929-94580249+ & hsa-miR-6507-5p & hsa-miR-5571-5p & hsa-miR-3927-3p & hsa-miR-3190-5p & hsa-miR-4529-5p \\
chr2:179517185-179517463- & hsa-miR-128-1-5p & hsa-miR-6779-5p & hsa-miR-939-5p & hsa-miR-4266 & hsa-miR-765 \\
chr9:16435553-16437522- & hsa-miR-378g & hsa-miR-6779-5p & hsa-miR-4674 & hsa-miR-149-5p hsa-miR-92a-1-5p \\
chr22:36006931-36007153- & hsa-miR-103a-2-5p & hsa-miR-764 & hsa-miR-636 & hsa-miR-4659b-3p hsa-miR-767-5p \\
\hline
\end{tabular}

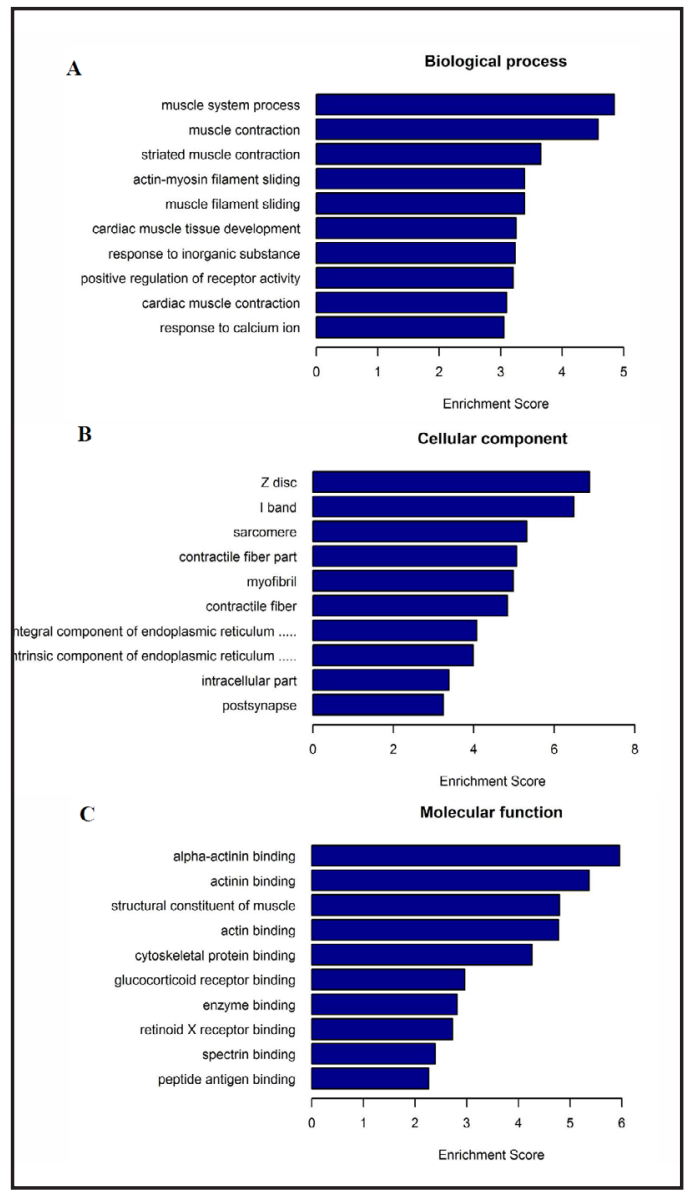

Fig. 5. Gene Ontology (GO) terms for differentially expressed circRNA genes. The bar plot presents the enrichment scores ( $-\log 10$ [P value]) of the top 10 significantly enriched GO terms: biological process (A), cellular component (B), and molecular function (C).

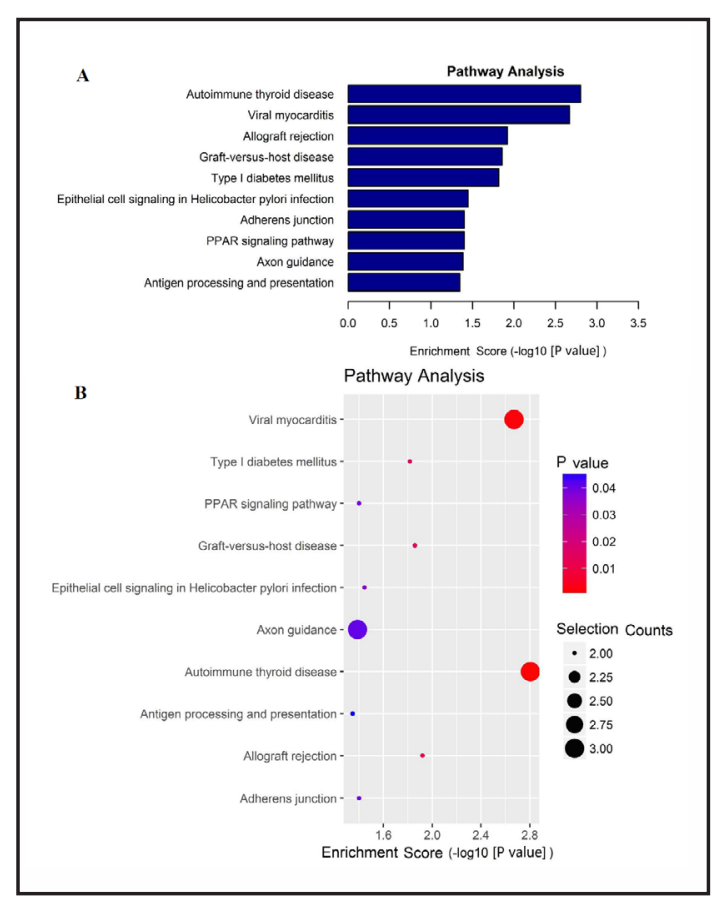

Fig. 6. Kyoto Encyclopedia of Genes and Genomes (KEGG) pathways of differentially expressed circRNA genes. The top 10 significantly enriched pathways and their scores ( $-\log 10$ [P value]) are listed as the $y$ axis and the $\mathrm{x}$ axis, respectively.

targeted mRNA network was also constructed (Fig. 8), in which each circRNA has five associated miRNAs and every miRNA has 10 predicted mRNA targets. The network indicates the potential competing endogenous RNA (ceRNA) relationships among circRNAs, miRNAs, and mRNAs in PTC. 


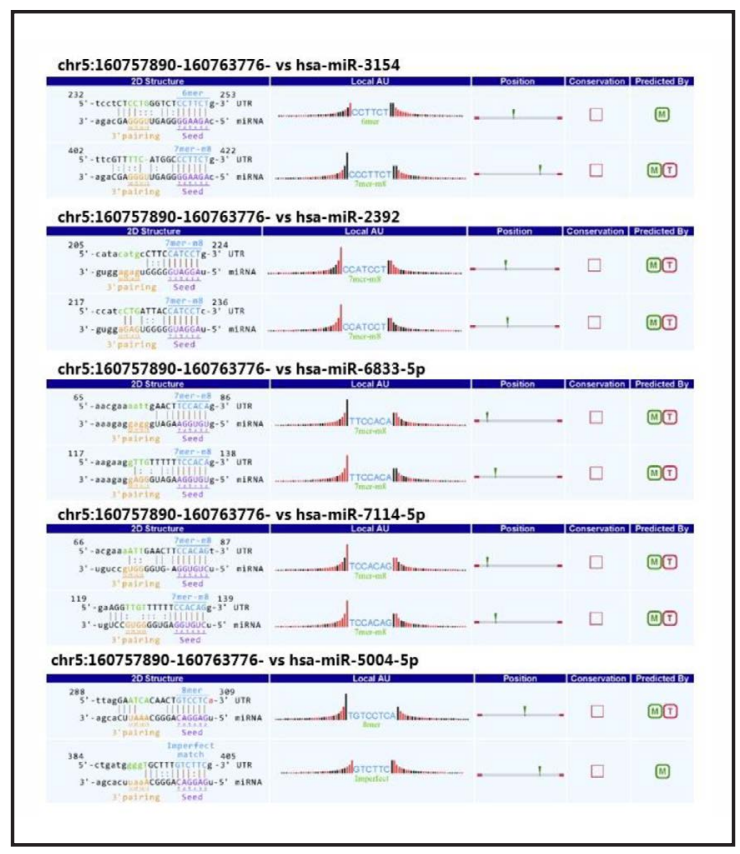

Fig. 7. Detailed annotation of circRNA-miRNA interactions between chr5:160757890-160763776and its target miRNAs.

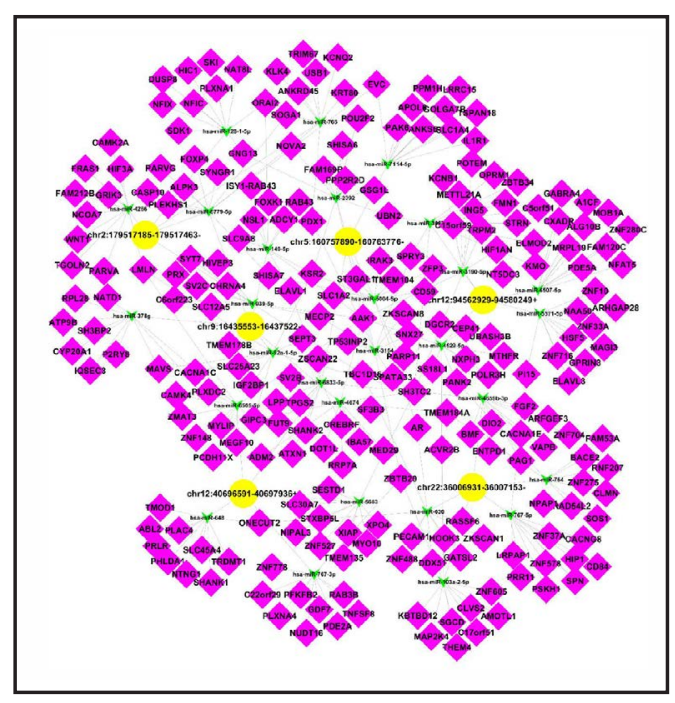

Fig. 8. Predicted circRNA-miRNA-mRNA network for the top three up- and downregulated circRNAs in PTC. Yellow represents circRNAs, green represents miRNAs, and pink represents miRNA target mRNAs.

\section{Discussion}

circRNAs were originally thought to be by-products of mRNA splicing errors [22]. However, recent studies have indicated that circRNAs are a stable, diverse, and conserved class of RNA molecules [7]. Compared with mRNAs, miRNAs, and lncRNAs, circRNAs are promising diagnostic and prognostic biomarkers because of their stability [23]. Owing to the advent of high-throughput RNA-Seq, numerous circRNAs have been identified as being dysregulated in certain cancers, such as gastric cancer [24], breast cancer [25], hepatocellular carcinoma [6, 26], pancreatic carcinoma [27], and esophageal carcinoma [28]; however, their expression statuses and roles in PTC remain to be determined. In this study, we used circRNA sequencing to obtain circRNA expression profiles for PTC tissues and paired adjacent normal tissues. Consequently, we identified 87 significantly differentially expressed circRNAs, among which chr5:160757890-160763776- was the most upregulated, and chr22:36006931-36007153the most downregulated in PTC. Among these differentially expressed circRNAs, we chose eight for qRT-PCR validation, the results of which were strongly consistent with those of circRNA sequencing, confirming the reliability of RNA-Seq. ROC curves also suggested that the validated circRNAs can serve as biomarkers for diagnosing PTC. Additionally, we identified 33 novel circRNAs from the RNA-Seq data. Although much work is needed, these novel circRNAs are specifically interesting for further study because of the lack of information about them in circRNA databases.

As circRNAs have been shown to regulate the function of their host mRNAs or parent genes [29], we attempted to explore the potential functions of circRNAs differentially expressed in PTC by analyzing their parent genes. GO and KEGG pathway analyses revealed that the parent genes are involved in certain pathways associated with cancer, such as the PPAR signaling pathway (related circRNAs and genes: chr19:4511523-4511918- and PLIN4; chr10:97154758-97170534- and SORBS1) and adherens junction (related circRNAs and genes: chr7:116339125-116340338+ and MET; chr10:97154758-97170534- and SORBS1). Previous studies have suggested that the PPAR signaling pathway contributes to thyroid cancer [30-32], and MET is a well-known proto-oncogene associated with multiple

\section{KARGER}


human cancers [33-35]. Therefore, circRNA chr19:4511523-4511918-, chr10:9715475897170534-, and chr7:116339125-116340338+ may participate in the tumorigenesis of PTC by regulating their parent genes.

circRNAs are closed continuous loops of RNA with multiple miRNA binding sites [4, 36], which enables them to sequester miRNAs and act as sponges of miRNA that regulate expression of target miRNAs [37, 38]. For example, the circRNA CiRS-7 contains more than 70 binding sites for miR-7 and can function as a sponge of this miRNA [5, 39]. Due to their abundance and stability, circRNAs are more effective in sponging miRNA than are other types of RNAs [40-44]. We also predicted the potential miRNA targets of circRNAs and constructed a circRNA-miRNA-mRNA network to explore the function of dysregulated circRNAs in PTC. The network illustrates the potential ceRNA relationships among circRNAs, miRNAs, and mRNAs. For example, chr5:160757890-160763776-, chr12:40696591-40697936+, and chr12:94562929-94580249+, as well as chr22:36006931-36007153-, chr9:1643555316437522-, and chr2:179517185-179517463-, are the top three up- and downregulated circRNAs and possibly regulate expression of certain mRNAs by binding to their target miRNAs. Dysregulated circRNAs may participate in the pathogenesis of PTC by regulating miRNAs and their target mRNAs. Future studies should focus on these circRNAs and their related miRNAs and mRNAs.

Several limitations should be acknowledged in the present study. First, only three pairs of samples were chosen for circRNA sequencing. The sample size for RNA-Seq is relatively small and thus some selection bias may exist. Second, the prognostic value of the circRNAs was not investigated in the present study due to the relatively short-term follow-up. Therefore, future studies with large sample sizes and long-term follow-up are still needed to verify our findings.

In conclusion, our study revealed a landscape of circRNA expression profiles in PTC. Numerous circRNAs were found to be dysregulated in PTC tissues compared with adjacent normal tissues. Our RNA-Seq and qRT-PCR results were strongly consistent. Bioinformatic analysis predicted the potential functions related to these circRNAs as well as several potential circRNA-miRNA-mRNA ceRNA-regulating networks. Additionally, the findings provide potential biomarkers for PTC. Further functional and mechanistic studies of these circRNAs will improve our understanding of tumorigenesis in PTC.

\section{Acknowledgements}

This work was supported by the National Natural Science Foundation of China (Grant Nos. 81702645, 81672642, 81702653, and 81602349) and Medical and Health Research Program of Zhejiang Province (Grant No. 2015DTA003).

\section{Disclosure Statement}

The authors declare to have no conflict of interests.

\section{References}

1 Siegel RL, Miller KD, Jemal A: Cancer statistics, 2016 CA: A Cancer Journal for Clinicians 2016;66:7-30.

2 The Cancer Genome Atlas Research Network: Integrated genomic characterization of papillary thyroid carcinoma. Cell 2014;159:676-690.

-3 Haugen BR, Alexander EK, Bible KC, Doherty GM, Mandel SJ, Nikiforov YE, Pacini F, Randolph GW, Sawka AM, Schlumberger M, Schuff KG, Sherman SI, Sosa JA, Steward DL, Tuttle RM, Wartofsky L: 2015 American Thyroid Association Management Guidelines for Adult Patients with Thyroid Nodules and Differentiated Thyroid Cancer: The American Thyroid Association Guidelines Task Force on Thyroid Nodules and Differentiated Thyroid Cancer. Thyroid 2016;26:1-133. 


\section{Cellular Physiology Cell Physiol Biochem 2018;47:1122-1132 \begin{tabular}{l|l} 
DOI: 10.1159/000490188 & $\begin{array}{l}\text { O 2018 The Author(s). Published by S. Karger AG, Basel } \\
\text { www.karger.com/cpb }\end{array}$
\end{tabular}}

Lan et al.: Circular RNA Expression Profiles in Papillary Thyroid Carcinoma

4 Chen LL, Yang L: Regulation of circRNA biogenesis. RNA Biol 2015;12:381-388.

5 Hansen TB, Jensen TI, Clausen BH, Bramsen JB, Finsen B, Damgaard CK, Kjems J: Natural RNA circles function as efficient microRNA sponges. Nature 2013;495:384-388.

6 Han D, Li J, Wang H, Su X, Hou J, Gu Y, Qian C, Lin Y, Liu X, Huang M, Li N, Zhou W, Yu Y, Cao X: Circular RNA circMTO1 acts as the sponge of microRNA-9 to suppress hepatocellular carcinoma progression. Hepatology 2017;66:1151-1164.

7 Memczak S, Jens M, Elefsinioti A, Torti F, Krueger J, Rybak A, Maier L, Mackowiak SD, Gregersen LH, Munschauer M, Loewer A, Ziebold U, Landthaler M, Kocks C, le Noble F, Rajewsky N: Circular RNAs are a large class of animal RNAs with regulatory potency. Nature 2013;495:333-338.

-8 Holdt LM, Stahringer A, Sass K, Pichler G, Kulak NA, Wilfert W, Kohlmaier A, Herbst A, Northoff BH, Nicolaou A, Gabel G, Beutner F, Scholz M, Thiery J, Musunuru K, Krohn K, Mann M, Teupser D: Circular noncoding RNA ANRIL modulates ribosomal RNA maturation and atherosclerosis in humans. Nat Commun 2016;7:12429.

9 Chen Y, Li C, Tan C, Liu X: Circular RNAs: a new frontier in the study of human diseases. J Med Genet 2016;53:359-365.

10 Kumar L, Shamsuzzama, Haque R, Baghel T, Nazir A: Circular RNAs: the emerging class of non-coding RNAs and their potential role in human neurodegenerative diseases. Mol Neurobiol DOI: 10.1007/s12035-0160213-8.

11 Xin Z, Ma Q, Ren S, Wang G, Li F: The understanding of circular RNAs as special triggers in carcinogenesis. Brief Funct Genomics 2017;16:80-86.

12 Kulcheski FR, Christoff AP, Margis R: Circular RNAs are miRNA sponges and can be used as a new class of biomarker. J Biotechnol 2016;238:42-51.

13 Zhang X, Yan Y, Lei X, Li A, Zhang H, Dai Z, Li X, Chen W, Lin W, Chen F, Ma J, Xie Q: Circular RNA alterations are involved in resistance to avian leukosis virus subgroup-J-induced tumor formation in chickens. Oncotarget 2017;8:34961-34970.

14 Dobin A, Davis CA, Schlesinger F, Drenkow J, Zaleski C, Jha S, Batut P, Chaisson M, Gingeras TR: STAR: ultrafast universal RNA-seq aligner. Bioinformatics 2013;29:15-21.

15 Cheng J, Metge F, Dieterich C: Specific identification and quantification of circular RNAs from sequencing data. Bioinformatics 2016;32:1094-1096.

16 Glazar P, Papavasileiou P, Rajewsky N: Circbase: a database for circular RNAs. RNA 2014;20:1666-1670.

17 Ghosal S, Das S, Sen R, Basak P, Chakrabarti J: Circ2traits: a comprehensive database for circular RNA potentially associated with disease and traits. Front Genet 2013;4:283.

18 Robinson MD, McCarthy DJ, Smyth GK: Edger: a bioconductor package for differential expression analysis of digital gene expression data. Bioinformatics 2010;26:139-140.

19 Friedman RC, Farh KK, Burge CB, Bartel DP: Most mammalian mRNAs are conserved targets of microRNAs. Genome Res 2009;19:92-105.

-20 Betel D, Koppal A, Agius P, Sander C, Leslie C: Comprehensive modeling of microRNA targets predicts functional non-conserved and non-canonical sites. Genome Biol 2010;11:R90.

-21 Smoot ME, Ono K, Ruscheinski J, Wang PL, Ideker T: Cytoscape 2.8: new features for data integration and network visualization. Bioinformatics 2011;27:431-432.

22 Qu S, Yang X, Li X, Wang J, Gao Y, Shang R, Sun W, Dou K, Li H: Circular RNA: a new star of noncoding RNAs. Cancer Lett 2015;365:141-148.

23 Zhuang ZG, Zhang JA, Luo HL, Liu GB, Lu YB, Ge NH, Zheng BY, Li RX, Chen C, Wang X, Liu YQ Liu FH, Zhou Y, Cai XZ, Chen ZW, Xu JF: The circular RNA of peripheral blood mononuclear cells: hsa_circ_0005836 as a new diagnostic biomarker and therapeutic target of active pulmonary tuberculosis. Mol Immunol 2017;90:264272.

24 Zhang J, Liu H, Hou L, Wang G, Zhang R, Huang Y, Chen X, Zhu J: Circular RNA_LARP4 inhibits cell proliferation and invasion of gastric cancer by sponging miR-424-5p and regulating LATS1 expression. Mol Cancer 2017;16:151.

25 Liang HF, Zhang XZ, Liu BG, Jia GT, Li WL: Circular RNA circ-ABCB10 promotes breast cancer proliferation and progression through sponging miR-1271. Am J Cancer Res 2017;7:1566-1576.

-26 Xue Y, Xiong Q, Wu Y, Li S, Ge F: Quantitative proteomics reveals the regulatory networks of circular RNA cdr1as in hepatocellular carcinoma cells. J Proteome Res 2017;16:3891-3902. 


\section{Cellular Physiology Cell Physiol Biochem 2018;47:1122-1132 \begin{tabular}{l|l|l} 
DOI: 10.1159/000490188 & O 2018 The Author(s). Published by S. Karger AG, Basel \\
www.karger.com/cpb
\end{tabular}}

Lan et al.: Circular RNA Expression Profiles in Papillary Thyroid Carcinoma

27 Li H, Hao X, Wang H, Liu Z, He Y, Pu M, Zhang H, Yu H, Duan J, Qu S: Circular RNA Expression Profile of Pancreatic Ductal Adenocarcinoma Revealed by Microarray. Cell Physiol Biochem 2016;40:1334-1344.

-28 Xia W, Qiu M, Chen R, Wang S, Leng X, Wang J, Xu Y, Hu J, Dong G, Xu PL, Yin R: Circular RNA has_ circ_0067934 is upregulated in esophageal squamous cell carcinoma and promoted proliferation. Sci Rep 2016;6:35576.

29 Ashwal-Fluss R, Meyer M, Pamudurti NR, Ivanov A, Bartok O, Hanan M, Evantal N, Memczak S, Rajewsky N, Kadener S: CircRNA biogenesis competes with pre-mRNA splicing. Mol Cell 2014;56:55-66.

30 Chu R, van Hasselt A, Vlantis AC, Ng EK, Liu SY, Fan MD, Ng SK, Chan AB, Liu Z, Li XY, Chen GG: The crosstalk between estrogen receptor and peroxisome proliferator-activated receptor gamma in thyroid cancer. Cancer-Am Cancer Soc 2014;120:142-153.

-31 Nikiforova MN, Nikiforov YE: Molecular diagnostics and predictors in thyroid cancer. Thyroid 2009;19:1351-1361.

-32 Zeng L, Geng Y, Tretiakova M, Yu X, Sicinski P, Kroll TG: Peroxisome proliferator-activated receptor-delta induces cell proliferation by a cyclin E1-dependent mechanism and is up-regulated in thyroid tumors. Cancer Res 2008;68:6578-6586.

33 Sterlacci W, Fiegl M, Gugger M, Bubendorf L, Savic S, Tzankov A: MET overexpression and gene amplification: prevalence, clinico-pathological characteristics and prognostic significance in a large cohort of patients with surgically resected nsclc. Virchows Arch 2017;471:49-55.

34 Kim WY, Shim SH, Jung HY, Dong M, Kim SN, Lee SJ: The gene copy number of c-MET has a significant impact on progression-free survival in Korean patients with ovarian carcinoma. Hum Pathol 2017;64:98105.

-35 Salgia R: MET in Lung Cancer: Biomarker Selection Based on Scientific Rationale. Mol Cancer Ther 2017;16:555-565.

-36 Huang C, Shan G: What happens at or after transcription: insights into circRNA biogenesis and function. Transcription 2015;6:61-64.

-37 Sand M, Bechara FG, Sand D, Gambichler T, Hahn SA, Bromba M, Stockfleth E, Hessam S: Circular RNA expression in basal cell carcinoma. Epigenomics-Uk 2016;8:619-632.

-38 Ahmed I, Karedath T, Andrews SS, Al-Azwani IK, Mohamoud YA, Querleu D, Rafii A, Malek JA: Altered expression pattern of circular RNAs in primary and metastatic sites of epithelial ovarian carcinoma. Oncotarget 2016;7:36366-36381.

39 Hansen TB, Kjems J, Damgaard CK: Circular RNA and miR-7 in cancer. Cancer Res 2013;73:5609-5612.

40 Du WW, Yang W, Liu E, Yang Z, Dhaliwal P, Yang BB: Foxo3 circular RNA retards cell cycle progression via forming ternary complexes with p21 and CDK2. Nucleic Acids Res 2016;44:2846-2858.

41 Jeck WR, Sharpless NE: Detecting and characterizing circular RNAs. Nat Biotechnol 2014;32:453-461.

42 Hansen TB, Jensen TI, Clausen BH, Bramsen JB, Finsen B, Damgaard CK, Kjems J: Natural RNA circles function as efficient microRNA sponges. Nature 2013;495:384-388.

43 Zhao J, Li L, Wang Q, Han H, Zhan Q Xu M: CircRNA expression profile in Early-Stage lung adenocarcinoma patients. Cell Physiol Biochem 2017;44:2138-2146.

44 Liu W, Zhang J, Zou C, Xie X, Wang Y, Wang B, Zhao Z, Tu J, Wang X, Li H, Shen J, Yin J: Microarray expression profile and functional analysis of circular RNAs in osteosarcoma. Cell Physiol Biochem 2017;43:969-985. 